\title{
Racial Disparities in Sustaining Breastfeeding in a Baby-Friendly Designated Southeastern United States Hospital: An Opportunity to Investigate Systemic Racism
}

\author{
Shauna Hemingway, ${ }^{1}$ Zaneta Forson-Dare, ${ }^{2}$ Myla Ebeling, ${ }^{3}$ and Sarah N. Taylor ${ }^{2}$
}

\begin{abstract}
Background: Racial disparities in breastfeeding rates persist in the United States with Black women having the lowest rates of initiation and continuation. A literature review attributes this to many factors-historical roles, cultural norms, lack of social support, and systemic racism in the health care and lactation support system. The Baby-Friendly Hospital Initiative (BFHI) is an evidence-based program to increase breastfeeding through standardized protocols, and, in a Southeastern U.S. academic center, it was associated with increased breastfeeding, but the effect on racial disparities in breastfeeding was unknown.

Methods: Through an institutional Perinatal Information Systems database, breastfeeding rates were compared before and after BFHI implementation. Breastfeeding initiation, sustained breastfeeding 24-hours before discharge, and patient demographics were assessed.

Results: After BFHI implementation, mothers were overall 1.17 (95\% confidence interval: 1.13-1.19) times more likely to initiate breastfeeding. For Black mothers, breastfeeding initiation increased significantly from $52 \%$ to $66 \%$, but they were significantly less likely to sustain in-hospital breastfeeding compared to nonBlack mothers $(69.4 \%$ versus $84.6 \%, p<0.0001)$. Several demographic and medical comorbidities were significantly associated with failure to sustain breastfeeding to hospital discharge. When controlling for these factors, the racial disparity persisted.

Conclusions: Since BFHI implementation, the racial gap in breastfeeding initiation decreased, but a significant disparity remained for sustained in-hospital breastfeeding. This study highlights the success of the BFHI program, but in the context of the current literature, also reveals the need for further work to ensure interventions are culturally competent and delivered equitably to support sustained breastfeeding for Black women.
\end{abstract}

Keywords: Baby-Friendly Hospital Initiative, breastfeeding, Black, Southeastern, race, systemic racism

\section{Introduction}

$\mathbf{R}$ ACIAL DISPARITY IN BREASTFEEDING in the United States has been explained by a number of different factors, including the historical role of Black women forced to breast feed the white infants first for their slave masters and continuing through the Jim Crow era. ${ }^{1}$ Other factors include lack of cultural normalization of breastfeeding in the Black community, lack of support from family members and the community, ${ }^{2}$ financial pressures necessitating quicker return to work, shorter maternity leaves which decreases the duration of breastfeeding, ${ }^{3}$ and holding jobs that do not provide breastfeeding support in terms of adequate break time and safe, clean, and private environments to pump breast milk. ${ }^{4}$

Another potential barrier for Black women to breastfeed in the United States is systemic racism. Systemic racism in the United States health care system is associated with adverse

\footnotetext{
${ }^{1}$ Medical University of South Carolina Department of Obstetrics and Gynecology, Charleston, South Carolina, USA.

${ }^{2}$ Yale School of Medicine Department of Pediatrics, New Haven, Connecticut, USA.

${ }^{3}$ Medical University of South Carolina Department of Pediatrics, Charleston, South Carolina, USA.
} 
health outcomes for Blacks. ${ }^{5}$ When implementing a community or hospital-based intervention to improve health care, it is important to ensure that the intervention resolves rather than perpetuates systemic inequities.

The Baby-Friendly Hospital Initiative (BFHI) is a hospitalbased intervention launched in 1991 by the World Health Organization (WHO) and United Nations International Children's Fund to improve breastfeeding initiation, duration, and exclusivity rates. ${ }^{6} \mathrm{BFHI}$ is associated with improved rates of breastfeeding outcomes, including a $20 \%$ increased likelihood of early initiation of breastfeeding, $49 \%$ increase in exclusive breastfeeding for $0-5$ months, and $66 \%$ increase in any breastfeeding up to 6 months as demonstrated by a published meta-analysis. ${ }^{7}$

Breastfeeding is known to be beneficial for both infants and mothers, ${ }^{8}$ and within the United States, there are racial disparities in the rates of breastfeeding with non-Hispanic Black (NHB) infants being breastfed less often than other racial and ethnic groups. ${ }^{9,10}$ In estimates of disease burden, suboptimal breastfeeding is associated with a 2.2 times increase in excess child deaths for the NHB compared to the non-Hispanic white population. ${ }^{11}$ This risk is even more significant given that Black infants have twice the infant mortality compared to white infants at $11 \%$ and $5 \%$, respectively. ${ }^{12,13}$ In terms of maternal benefits, breastfeeding has been shown to help decrease the risk of endometrial and breast cancers, for which Black women suffer more aggressive forms. ${ }^{14,15}$ In addition, breastfeeding also has been linked to more rapid return to prepregnancy weight and better glycemic control. This association is significant for Black women in the United States, as they are more likely to suffer from higher rates of obesity and diabetes and their associated sequelae. ${ }^{16-18}$ Improving breastfeeding rates starts with initiation of breastfeeding, but the continuation of breastfeeding is associated with the most benefit.

In the United States, Healthy People 2020 included breastfeeding goals to increase both initiation and continuation of breastfeeding through the first year. The Healthy People 2020 breastfeeding goals were largely met with $84 \%$ of infants born in 2017 "ever breastfed" compared to the goal of $81 \% .{ }^{19}$ Although Black non-Hispanic infants in the United States continue to be breastfed less than any other racial or ethnic group, with $73.7 \%$ ever breastfeeding and $47.8 \%$ breastfeeding at 6 months in 2017, Black infant breastfeeding rates are higher in 2017 compared to 20112015. 9,10 This increase in Black non-Hispanic breastfeeding rates may be related to the growing number of U.S. hospitals adopting the BFHI guidelines, which require all pregnant women be informed about the benefits of breastfeeding and all breastfeeding women receive in-hospital support, which is a first step in providing equitable health care.

Data regarding BHFI implementation in U.S. hospitals serving disadvantaged populations have been collected to evaluate its impact. ${ }^{20-22}$ One study, conducted in Boston, Massachusetts, evaluating outcomes specific to the African American population ${ }^{23}$ found that Black women had improved breastfeeding initiation rates (defined as any breastfeeding in the hospital) from $35 \%$ to $74 \%$ after BFHI implementation. While implementation of BFHI did show improvement in initiation rates, this study did not address whether these mothers sustained breastfeeding to hospital discharge, which is impor- tant in predicting continued breastfeeding after discharge. In addition, this study did not discuss factors associated with initiation rates, which can act as predictors for women at highest risk for not breastfeeding.

Therefore, the primary aim of this study was to determine racial differences in breastfeeding initiation and breastfeeding sustained through birth hospitalization rates after BabyFriendly USA ${ }^{\circledR}$ hospital designation at a Southeastern United States Hospital. The secondary aim was to evaluate the demographics and medical comorbidities associated with sustained breastfeeding.

\section{Materials and Methods}

A retrospective, cohort study was conducted with data available from the Perinatal Information Systems Database at a single, academic institution serving the urban, suburban, and rural Southeastern portion of South Carolina. This research study was IRB-approved by the Medical University of South Carolina. Study subjects were all women who delivered an infant or infants who were admitted directly to the wellnewborn service and remained on this service throughout hospitalization. To be admitted to the well-newborn service, an infant must be $\geq 350 / 7$ weeks' postmenstrual age and able to eat without gavage or IV fluid requirements. January 2010 through December 2011 was defined as "preBFHI" and January 2014 through December 2015 as "postBFHI." The specific dates were chosen because the institutioninitiated work to achieve Baby-Friendly Hospital Designation (Baby-Friendly USA) in 2012 with designation achieved September 2013.

Breastfeeding initiation was defined as any breastfeeding during infant born at hospitalization. Sustained breastfeeding was defined as any breastfeeding and breastfeeding in the 24 hours before maternal hospital discharge. Maternal demographics collected from the medical record included race, insurance status, marital status, multiple pregnancy, preterm delivery, mode of delivery, small-for-gestational age infant, and maternal disease such as preeclampsia, diabetes mellitus, chronic hypertension, and obesity. For this study, maternal race was categorized as Black or non-Black. Analysis included chi-square and Fisher exact tests with relative risk calculation at a $95 \%$ confidence interval. Logistic regression model was used to adjust for potential confounders to breastfeeding.

\section{Results}

The number of mothers who delivered newborns admitted to the newborn service was 3079 between 2010 and 2011 and 3606 between 2014 and 2015. Maternal demographics are compared between the two groups and are provided in Table 1. There were statistically significant differences as it relates to marital status, cesarean section rates, private insurance, substance abuse, and chronic hypertension for mothers pre- and post-BFHI. There were also increased NHW subjects and decreased Hispanic and NHB mothers post-BFHI.

Breastfeeding initiation rates are provided in Table 2. Post-BFHI, mothers were significantly more likely to initiate breastfeeding when compared to mothers who delivered at the institution pre-BFHI. A significant increase in breastfeeding initiation post-BFHI was seen for both the nonBlack and Black mothers. The relative improvement in this 
Table 1. Demographics of the Pre-Baby-Friendly Hospital Initiative and Post-Baby-Friendly Hospital INITIATIVE COHORTS

\begin{tabular}{lcc}
\hline & $\begin{array}{c}\text { Pre-BFHI } \\
\mathrm{n}=3079\end{array}$ & $\begin{array}{r}\text { Post-BFHI } \\
\mathrm{n}=3606\end{array}$ \\
\hline C-section* & $29 \%$ & $31 \%$ \\
Black non-Hispanic* & $42 \%$ & $38 \%$ \\
White non-Hispanic* & $32 \%$ & $44 \%$ \\
Hispanic* & $24 \%$ & $16 \%$ \\
Other ethnicity/race & $2 \%$ & $2 \%$ \\
Multiples & $2 \%$ & $2 \%$ \\
Married* & $38 \%$ & $51 \%$ \\
Private insurance* & $23 \%$ & $61 \%$ \\
Preterm birth & $6 \%$ & $7 \%$ \\
Preeclampsia & $5 \%$ & $4 \%$ \\
Diabetes & $2 \%$ & $2 \%$ \\
Chronic hypertension* & $6 \%$ & $8 \%$ \\
Obesity & $8 \%$ & $8 \%$ \\
Substance abuse* & $3 \%$ & $4 \%$ \\
Mean age (years) & 27 & 28 \\
Average length of stay (days) & 2 & 2 \\
\hline
\end{tabular}

Significant difference $(p$-value $<0.05)$ denoted by $*$. BFHI, Baby-Friendly Hospital Initiative.

population is 1.1 times the pre-BFHI rate. For the Black mothers, the relative improvement in this population is 1.27 times the pre-BFHI rate, which is higher than the relative improvement in the non-Black population.

Post-BFHI, $80 \%$ of the mothers who initiated breastfeeding were still breastfeeding in the 24 hours before hospital discharge. The median hospital stay for both pre- and postBFHI was 2 days. When stratified by race, $84.6 \%$ of non-Black breastfeeding-initiating mothers sustained breastfeeding, while only $69.5 \%$ of Black breastfeeding-initiating mothers sustained breastfeeding $(p<0.0001)$. Black mothers were 2.4 times less likely to sustain breastfeeding than non-Black mothers. Other factors such as receiving prenatal care outside of an MUSC clinic, unmarried status, absence of private insurance, younger age, and diseases such as obesity, diabetes mellitus, preeclampsia, chronic hypertension, substance abuse, preterm delivery, and small-for-gestational age infant also were all significantly associated with failure to sustain breastfeeding (Table 3). However, when controlling for these factors, the racial disparity persisted.

\section{Discussion}

The BFHI has been expanding throughout the United States as evidenced by $3 \%$ of all U.S. births occurring in

Table 2. Breastfeeding Initiation by Race FOR COMPARED BETWEEN BIRTHS

Pre- And Post-Baby-Friendly Hospital Initiative

\begin{tabular}{lccr}
\hline $\begin{array}{l}\text { Initiation } \\
\text { of breastfeeding }\end{array}$ & $\begin{array}{c}\text { Pre- } \\
\text { BFHI } \\
\mathrm{n}=3079\end{array}$ & $\begin{array}{c}\text { Post- } \\
\text { BFHI } \\
\mathrm{n}=3606\end{array}$ & $\begin{array}{c}\text { Increased } \\
\text { likelihood } \\
(95 \% \text { CI })\end{array}$ \\
\hline All mothers & $69 \%$ & $81 \%$ & $1.17(1.13-1.19)$ \\
Black mothers & $52 \%$ & $66 \%$ & $1.27(1.19-1.35)$ \\
Non-Black mothers & $81 \%$ & $90 \%$ & $1.1(1.07-1.13)$
\end{tabular}

CI, confidence interval.
Baby-Friendly Hospitals in 2007 versus $20 \%$ in 2017. In the United States, Black women have the lowest rates of breastfeeding in the setting of high rates of poor pregnancy and obstetrical outcomes such as preterm birth, low birth rate infants, and preeclampsia. ${ }^{24,25}$ In this hospital with adoption of the BFHI, institutional breastfeeding rates improved, and the rates of breastfeeding initiation improved more significantly for Black women compared to non-Black women. However, although the gap for breastfeeding initiation among different racial groups was narrowing, Black women still faced significant disparities in terms of breastfeeding initiation and continuation.

In research aimed to identify why this racial disparity persists, individual factors such as "negative attitudes" from the Black community as it relates to breastfeeding and sexuality, lack of role models, as well as disproportionate exposure to formula feeding versus breastfeeding are hypothesized to lead to Black women feeling more comfortable with formulafeeding rather than breastfeeding. ${ }^{26}$ However, across multiple qualitative studies, Black women self-identify breastfeeding to be the healthiest and most desired feeding method for their infants. ${ }^{27-31}$ In addition, socioeconomic status (SES) is an influential intersecting factor in the health disparities between Black and non-Black women and their infants, but racial disparities persist, even in Black women with high SES. Black women with advanced degrees have worse obstetric and neonatal outcomes than do non-Black women of low SES or those without a high school diploma, ${ }^{24}$ suggesting race to be the consistent influential factor. Focusing on individual factors rather than the systemic inequities such as racism in the social and health care infrastructure leads to ineffective interventions that are centered on external factors beyond the individual's control. ${ }^{32}$ When instead attention is paid to the history of Black people receiving substandard or reduced care in the United States, opportunities to rectify these disparities are apparent, and institutionalized racism and its deleterious effects on health outcomes can be eliminated. ${ }^{5,33}$

This research study showed that, despite an improvement in initiation, Black mothers were less likely to sustain breastfeeding to hospital discharge necessitating an examination of the factors in health care provision that could have caused this attrition. International Board-Certified Lactation Consultants (IBCLC) have been shown to improve breastfeeding initiation in women overall, including in minority women. ${ }^{34-36}$ While these initiatives have proved beneficial, they have not lead to a closing of the racial gap in breastfeeding. Thomas (2018) interviewed 36 IBCLC with the intention to investigate "race-based discrimination against patients in the course of lactation care.",37 They reported inherent presumptions that women of color will not breastfeed and so were given fewer lactation referrals, deprioritized in work flow, and received reduced attention and support. They also reported overt racist comments made by colleagues and increased referrals of Black mothers to social work and long-acting birth control. This disparity in treatment is concerning and likely negatively affects the rates of breastfeeding among Black mothers. ${ }^{38}$

This is consistent with the literature that shows that care providers have more negative perceptions of Black women. ${ }^{39}$ Vedam et al. conducted a national study of women who received maternity care and found that women of color disproportionately reported receiving inequitable treatment, 
Table 3. In Mothers Post-Baby-Friendly Hospital Initiative, the Likelihood of Sustaining Breastfeeding as Related to Maternal Medical Comorbidities

\begin{tabular}{|c|c|c|c|}
\hline \multirow[b]{2}{*}{ Maternal diagnoses } & \multicolumn{2}{|c|}{$\begin{array}{c}\text { Mothers post-BFHI with } \\
\text { breastfeeding initiation } \\
\text { and not sustaining breastfeeding (\%) }\end{array}$} & \multirow{2}{*}{$\begin{array}{l}\text { Relative risk }(95 \% \text { CI) } \\
\text { that factor is present for mothers } \\
\text { who did not sustain breastfeeding }\end{array}$} \\
\hline & $\begin{array}{c}\text { Risk factor } \\
\text { Present }\end{array}$ & $\begin{array}{l}\text { Risk factor } \\
\text { Not present }\end{array}$ & \\
\hline Multiples & 51.5 & 19.3 & $2.66(2.09-3.40)$ \\
\hline Preeclampsia & 41.9 & 19.3 & $2.16(1.69-2.78)$ \\
\hline Diabetes mellitus & 41.9 & 19.6 & $2.14(1.58-2.90)$ \\
\hline Preterm delivery & 39.8 & 60.2 & $2.11(1.73-2.58)$ \\
\hline Unmarried & 28.1 & 13.9 & $2.02(1.74-2.35)$ \\
\hline Black race & 30.6 & 15.8 & $1.99(1.72-2.29)$ \\
\hline Chronic hypertension & 33.5 & 19.1 & $1.76(1.43-2.17)$ \\
\hline Nonprivate insurance & 27.6 & 15.8 & $1.7(1.51-2.01)$ \\
\hline Obesity & 31.6 & 19.1 & $1.65(1.33-2.04)$ \\
\hline Infant small for gestational age & 26.8 & 19.6 & $1.37(1.07-1.75)$ \\
\hline
\end{tabular}

being ignored by their provider or being shouted at by their provider compared with white women. ${ }^{31}$ Another review article of 37 studies found Black women received little breastfeeding advice or encouragement and provider's assumptions that they would not breastfeed led to differences in the care provided to them compared with other races. ${ }^{40}$

There are a dearth of Black lactation consultants, and it is increasingly recognized that they face restrictions in accessing the IBCLC credentials and gaining employment ${ }^{41,42}$ making them less available. They serve as an essential resource as role models to provide culturally competent care with reduced effects of racial implicit bias. ${ }^{37}$ Geographic disparities exist in lactation support coverage with less coverage in areas with high Black populations, ${ }^{43}$ and Black women report inconsistent lactation support, some citing that they were even discouraged from breastfeeding. ${ }^{44} \mathrm{~A}$ national survey, Listening to Mothers, showed that Black women were more likely to be offered formula than nonBlack women. $^{45}$

Providers across the health professions train and work within a societal construct of systemic and structural racism, and patients live, work, and are affected by this same overarching system. Thus, to rectify disparities as they pertain to race, the inequities in breastfeeding need to be approached as an issue of social justice rather than addressed simply from a medical perspective. ${ }^{46}$ Especially when planning interventions, it must be noted that "service availability is not synonymous with service accessibility." 32 Racism, both cultural and institutional, provides both immediate stress and restrict access and resources essential to successful breastfeeding. ${ }^{27}$ It is the key to identify the structural and institutional roots of the individual bias of health care providers to effectively address it, ${ }^{47}$ which will in turn reduce racial disparities in breastfeeding.

A limitation of this study is that it is a retrospective comparison. Potential confounders such as community or media influence were not evaluated. The study is also limited by the lack of data to determine mother's plan to exclusive breastfeeding or to combination feed. This lack of comprehensive data in the medical record is common in breastfeeding research and limits the ability to truly assess the effectiveness of interventions. ${ }^{32}$ During this study, mothers were counseled to exclusively breastfeed through the birth hospitalization, and, therefore, combination feeding of both breastfeeding and formula was not given as a maternal milk choice at hospital admission. In addition, there was no documentation for the reason for formula feeding occurrence such as maternal choice versus medical indication. Finally, this study does not address posthospital discharge sustainability of breastfeeding through the first 1 to 2 years as recommended by the American Academy of Pediatrics ${ }^{8}$ and $\mathrm{WHO},{ }^{48}$ which is critical to achieving the maximal benefits of breastfeeding for both mother and baby.

\section{Conclusion}

In conclusion, an overall improvement in breastfeeding initiation was observed following Baby-Friendly Hospital Designation, with the greatest increase observed for Black women. However, the BFHI program failed to close the racial gap in sustaining breastfeeding to hospital discharge even when controlling for confounding factors. Sustaining breastfeeding to hospital discharge is a predictor for longterm breastfeeding exclusivity and duration. Explaining this finding in the context of the current literature identifies barriers within the health care and lactational support system due to structural racism and bias in health care delivery. Given the adverse health outcomes and loss of key protective benefits of breastfeeding, there is an urgency to addressing this disparity and a need to approach it through the lens of social justice. This study reveals the need for further work to ensure interventions designed to improve breastfeeding are culturally competent and delivered equitably to support sustained breastfeeding for Black women.

\section{Acknowledgments}

S.H. thanks both past and present Medical University of South Carolina residency research coordinators Dr. Lazenby, Dr. Young-Pierce, and Dr. Edenfield, who participated in development of this project. All authors appreciate the staff and families at the Medical University of South Carolina, whose participation in this study provides a foundation for all institutions to recognize inequity in outcomes and embrace opportunities to end racism. 


\section{Disclosure Statement}

No competing financial interests exist.

\section{Funding Information}

No funding was received for conduction of this study.

\section{References}

1. Obeng CS, Emetu RE, Curtis TJ. African-American Women's perceptions and experiences about breastfeeding. Front Public Health 2015;3:273.

2. Beauregard JL, Hamner HC, Chen J, et al. Racial disparities in breastfeeding initiation and duration among U.S. infants born in 2015. MMWR Morb Mortal Wkly Rep 2019; 68:745-748.

3. Chuang $\mathrm{CH}$, Chang PJ, Chen YC, et al. Maternal return to work and breastfeeding: A population-based cohort study. Int J Nurs Stud 2010;47:461-474.

4. Johnson AM, Kirk R, Muzik M. Overcoming workplace barriers: A focus group study exploring African American mothers' needs for workplace breastfeeding support. J Hum Lact 2015;31:425-433.

5. Feagin J, Bennefield Z. Systemic racism and U.S. health care. Soc Sci Med 2014;103:7-14.

6. Brodribb W, Kruske S, Miller YD. Baby-friendly hospital accreditation, in-hospital care practices, and breastfeeding. Pediatrics 2013;131:685-692.

7. Sinha B, Chowdhury R, Sankar MJ, et al. Interventions to improve breastfeeding outcomes: A systematic review and meta-analysis. Acta Paediatr 2015;104:114-134.

8. American Academy of Pediatrics. Breastfeeding and the use of human milk. Pediatrics 2012;129:e827-e841.

9. Anstey EH, Chen J, Elam-Evans LD, et al. Racial and geographic differences in breastfeeding-United States, 20112015. MMWR Morb Mortal Wkly Rep 2017;66:723-727.

10. Centers for Disease Control and Prevention. "Any" and "Exclusive" Breastfeeding Rates by Sociodemographics 2017. [accessed October 1, 2020]; Available at https://www.cdc.gov/ breastfeeding/data/nis_data/rates-any-exclusive-bf-socio-dem2017.html.

11. Bartick MC, Jegier BJ, Green BD, et al. Disparities in breastfeeding: Impact on maternal and child health outcomes and costs. J Pediatr 2017;181:49-55 e6.

12. Victora CG, Bahl R, Barros AJ, et al. Breastfeeding in the 21st century: Epidemiology, mechanisms, and lifelong effect. Lancet 2016;387:475-490.

13. Mathews TJ, Driscoll AK. Trends in infant mortality in the United States, 2005-2014. NCHS Data Brief 2017;279:1-8.

14. Islami F, Liu Y, Jemal A, et al. Breastfeeding and breast cancer risk by receptor status-A systematic review and meta-analysis. Ann Oncol 2015;26:2398-2407.

15. Ma X, Zhao LG, Sun JW, et al. Association between breastfeeding and risk of endometrial cancer: A metaanalysis of epidemiological studies. Eur J Cancer Prev 2018;27:144-151.

16. Palmer JR, Kipping-Ruane K, Wise LA, et al. Lactation in relation to long-term maternal weight gain in AfricanAmerican women. Am J Epidemiol 2015;181:932-939.

17. Schwarz EB, Ray RM, Stuebe AM, et al. Duration of lactation and risk factors for maternal cardiovascular disease. Obstet Gynecol 2009;113:974-982.

18. Stuebe AM, Schwarz EB, Grewen K, et al. Duration of lactation and incidence of maternal hypertension: A lon- gitudinal cohort study. Am J Epidemiol 2011;174:11471158.

19. Committee USB. Healthy People 2020. 2020 [accessed October 1, 2020]; Available at www.usbreastfeeding.org/p/ $\mathrm{cm} / \mathrm{ld} / \mathrm{fid}=221$.

20. Labbok MH, Taylor EC, Nickel NC. Implementing the ten steps to successful breastfeeding in multiple hospitals serving low-wealth patients in the US: Innovative research design and baseline findings. Int Breastfeed J 2013;8:5.

21. Munn AC, Newman SD, Mueller M, et al. The impact in the United States of the Baby-Friendly Hospital Initiative on early infant health and breastfeeding outcomes. Breastfeed Med 2016;11:222-230.

22. Munn AC, Newman SD, Phillips SM, et al. Factors Influencing Southeastern U.S. Mothers' Participation in BabyFriendly Practices: A Mixed-Methods Study. J Hum Lact 2018;34:821-834.

23. Philipp BL, Merewood A, Miller LW, et al. Baby-friendly hospital initiative improves breastfeeding initiation rates in a US hospital setting. Pediatrics 2001;108:677-681.

24. Collins JW, Jr., David RJ. Racial disparity in low birth weight and infant mortality. Clin Perinatol 2009;36:63-73.

25. Meeme A, Buga GA, Mammen M, et al. Angiogenic imbalance as a contributor to the pathophysiology of preeclampsia among black African women. J Matern Fetal Neonatal Med 2017;30:1335-1341.

26. Jones KM, Power ML, Queenan JT, et al. Racial and ethnic disparities in breastfeeding. Breastfeed Med 2015;10:186-196.

27. Griswold MK, Crawford SL, Perry DJ, et al. Experiences of racism and breastfeeding initiation and duration among first-time mothers of the Black Women's Health Study. J Racial Ethn Health Disparities 2018;5:1180-1191.

28. Schindler-Ruwisch J, Roess A, Robert RC, et al. Determinants of breastfeeding initiation and duration among African American DC WIC recipients: Perspectives of recent mothers. Womens Health Issues 2019;29:513-521.

29. Asiodu IV, Waters CM, Dailey DE, et al. Infant feeding decision-making and the influences of social support persons among first-time African American mothers. Matern Child Health J 2017;21:863-872.

30. Johnson AM, Kirk R, Rooks AJ, et al. Enhancing breastfeeding through healthcare support: Results from a focus group study of African American Mothers. Matern Child Health J 2016;20(Suppl 1):92-102.

31. Vedam S, Stoll K, Taiwo TK, et al. The giving voice to mothers study: inequity and mistreatment during pregnancy and childbirth in the United States. Reprod Health 2019; $16: 77$.

32. Reis-Reilly H, Fuller-Sankofa N, Tibbs C. Breastfeeding in the community: Addressing disparities through policy, systems, and environmental changes interventions. J Hum Lact 2018;34:262-271.

33. Noonan AS, Velasco-Mondragon HE, Wagner FA. Improving the health of African Americans in the USA: An overdue opportunity for social justice. Public Health Rev 2016;37:12.

34. Castrucci BC, Hoover KL, Lim S, et al. A comparison of breastfeeding rates in an urban birth cohort among women delivering infants at hospitals that employ and do not employ lactation consultants. J Public Health Manag Pract 2006;12:578-585.

35. Haase B, Brennan E, Wagner CL. Effectiveness of the IBCLC: Have we made an impact on the care of breast- 
feeding families over the past decade? J Hum Lact 2019;35: 441-452.

36. Patel S, Patel S. The effectiveness of lactation consultants and lactation counselors on breastfeeding outcomes. J Hum Lact 2016;32:530-541.

37. Thomas EV. "Why Even Bother; They Are Not Going to Do It?" The structural roots of racism and discrimination in lactation care. Qual Health Res 2018;28:1050-1064.

38. Odom EC, Li R, Scanlon KS, et al. Association of family and health care provider opinion on infant feeding with mother's breastfeeding decision. J Acad Nutr Diet 2014; 114:1203-1207.

39. Moskowitz D, Thom DH, Guzman D, et al. Is primary care providers' trust in socially marginalized patients affected by race? J Gen Intern Med 2011;26:846-851.

40. Spencer BS, Grassley JS. African American women and breastfeeding: An integrative literature review. Health Care Women Int 2013;34:607-625.

41. Payne SL. A call to action: Lactation equity through professional diversification. J Hum Lact 2014;30:396-397.

42. Mojab CG. Pandora's box is already open: Answering the ongoing call to dismantle institutional oppression in the field of breastfeeding. J Hum Lact 2015;31:32-35.

43. Evans K, Labbok M, Abrahams SW. WIC and breastfeeding support services: Does the mix of services offered vary with race and ethnicity? Breastfeed Med 2011;6:401-406.
44. DeVane-Johnson S, Woods-Giscombe C, Thoyre S, et al. Integrative literature review of factors related to breastfeeding in African American women: Evidence for a potential paradigm shift. J Hum Lact 2017;33:435-447.

45. Declercq E, Sakala C, Corry M, et al. Listening to Mothers: Pregnancy and Birth. New York Childbirth Connection, 2013.

46. Smith PH. Social justice at the core of breastfeeding protection, promotion and support: A conceptualization. J Hum Lact 2018;34:220-225.

47. Bowen Matthew D. Toward a structural theory of implicit racial and ethnic bias in health care. Health Matrix Clevel 2015;25:61-85.

48. W.H.O. Guideline: Counselling of Women to Improve Breastfeeding Practices. Geneva: World Health Organization, 2018.

Address correspondence to: Sarah N. Taylor, MD, MSCR

Yale School of Medicine Department of Pediatrics PO Box 208064

New Haven, CT 06520

USA

E-mail: sarah.n.taylor@yale.edu 